\title{
Optimal First-Line Treatment of Metastatic Renal-Cell Carcinoma: A Network Meta-Analysis
}

\author{
Andrew W. Hahn ${ }^{a}$, Peter Hale ${ }^{a}$, Benjamin L. Maughan ${ }^{a}$, \\ Benjamin Haaland ${ }^{\mathrm{b}, 1, *}$ and Neeraj Agarwal ${ }^{\mathrm{a}, 1, *}$ \\ ${ }^{a}$ Division of Medical Oncology, Department of Internal Medicine, Huntsman Cancer Institute, \\ University of Utah, Salt Lake City, UT, USA \\ ${ }^{\mathrm{b}}$ Department of Population Health Sciences, Huntsman Cancer Institute, University of Utah, \\ Salt Lake City, UT, USA
}

\begin{abstract}
.
Background: First-line treatment of metastatic renal cell carcinoma (mRCC) has incrementally evolved over the past decade. Most recently, promising clinical trials for first-line cabozantinib, nivolumab plus ipilimumab, and bevacizumab plus atezolizumab were reported. However, the comparator arm in all of these trials was sunitinib, so no head-to-head comparison exists for contemporary first-line treatments.

Objective: To provide an indirect comparison of first-line treatments for mRCC that are currently approved or likely soon to be approved.

Methods: Pivotal phase 2/3 clinical trials comparing currently approved first-line agents and novel regimens of nivolumab plus ipilimumab and bevacizumab plus atezolizumab were included if they reported overall survival (OS), progression-free survival (PFS), or objective response rate (ORR). Network meta-analysis of OS, PFS, and ORR was conducted in the context of Bayesian hierarchical log-linear models with both within and between study variance components.

Results: For OS, evidence suggests nivolumab plus ipilimumab may be the best first-line treatment available for mRCC (probability best 52\%). In contrast, cabozantinib may be superior to other first-line treatments for PFS (probability best $69 \%$ ) and ORR (probability best $95 \%$ ).

Conclusion: For first-line treatment of $\mathrm{mRCC}$, cabozantinib appears superior to other agents for PFS and ORR, whereas, nivolumab plus ipilimumab may be the best treatment for OS. No single agent was superior to comparators across all endpoints. First-line treatment for mRCC should be tailored to individual cases based on the unique characteristics of each treatment regimen.
\end{abstract}

Keywords: mRCC, treatment, first-line, meta-analysis, cabozantinib, nivolumab, atezolizumab

\footnotetext{
${ }^{1}$ Co-corresponding author.

*Correspondence to: Neeraj Agarwal, MD, Associate Professor of Medicine, Director, GU Medical Oncology, Huntsman Cancer Institute, University of Utah, 2000 Circle of Hope, Ste. 2123, Salt Lake City, UT 84112, USA. Tel.: +1 801585 0255; Fax: +1 801585 0124; E-mail: neeraj.agarwal@ hci.utah.edu and Benjamin Haaland, $\mathrm{PhD}$, Associate Professor, Division of Biostatistics, Department of Population Health Sciences, Huntsman Cancer Institute, University of Utah, 2000 Circle of Hope, Ste. 2123, Salt
}

\section{INTRODUCTION}

Kidney cancer is the eighth most common cancer in the United States, and most cases of kidney cancer are renal cell carcinoma (RCC) [1]. In newly diagnosed RCC, a quarter of patients will present

Lake City, UT 84112, USA. Tel.: +1 801585 0255; Fax: +1 801 5850124 E-mail: ben.haaland@hci.utah.edu. 
with metastatic RCC (mRCC), while an additional 20-40\% who initially present with localized disease will go on to develop $\mathrm{mRCC}[2,3]$. mRCC portends a poor prognosis with median overall survival (OS) less than three years [4]. In the 1990s, treatment of mRCC was limited to high dose interleukin-2 and interferonalfa. Both treatments had poor response rates and significant toxicities. Then, improved understanding of the molecular pathways involved in the pathogenesis of mRCC led to the development of novel first-line treatments, including VEGF tyrosine kinase inhibitors (TKIs) (sorafenib, sunitinib, pazopanib), a monoclonal antibody against VEGF (bevacizumab), and mTOR inhibitors (temsirolimus) [5-9]. These agents improved survival outcomes for patients with mRCC.

In the past two years, multi-target TKIs and immune checkpoint inhibitors have again changed first-line treatment for mRCC. Among multi-target TKIs, cabozantinib, a VEGF, MET, and AXL TKI, was approved for first-line treatment of $\mathrm{mRCC}$ on the basis of the CABOSUN clinical trial [10]. Combination therapies, including immune checkpoint inhibitors and VEGF targeted therapy, were the next evolution in first-line treatment. In CheckMate 214, the combination of nivolumab, a programmed death receptor-1 (PD-1) inhibitor, plus ipilimumab, a cytotoxic T lymphocyte antigen-4 (CTLA-4) inhibitor, was shown to improve survival for mRCC patients [11]. Then, IMmotion 151 found that bevacizumab plus atezolizumab, a programmed death receptor ligand -1 (PD-L1) inhibitor, also improves outcomes as first-line treatment of mRCC [12]. While clinicians and patients now have an abundance of first-line treatment options for mRCC, no head-to-head comparison exists between cabozantinib, nivolumab plus ipilimumab, and bevacizumab plus atezolizumab as all were compared with sunitinib. This leaves clinicians uncertain regarding which treatment will optimize outcomes for an individual patient. In this network meta-analysis, our objective is to provide an indirect comparison of all first-line treatments that are currently or likely soon to be approved for mRCC.

\section{METHODS}

Pivotal phase $2 / 3$ clinical trials of currently or likely soon to be approved first-line treatments for mRCC, comparing nivolumab plus ipilimumab, cabozantinib, sunitinib, pazopanib, bevacizumab plus interferon, or temsirolimus to interferon, placebo, or one another, were included for metaanalysis if they reported OS, progression-free survival (PFS), or objective response rates (ORR) in patients with $\mathrm{mRCC}$ who had not received previous systemic treatment aside from cytokines. For all clinical trials included, the intention-to-treat cohort was used to provide a comparison across clinical trials.

Meta-analysis of OS, PFS, and ORR was conducted in the context of Bayesian hierarchical log-linear models with both within and between study variance components. In particular, the three relative efficacies which could potentially be reported within each trial (hazard ratios for OS and PFS, and odds ratios ORR) were modeled as multivariate Gaussian on the logarithm scale with an unstructured covariance with separate correlations for each pair of efficacy measures and separate marginal variances for each of the three relative efficacies, each inflated to ensure a fixed intra-cluster correlation between relative efficacies in the same study. Therapies which achieve the combined benchmarks (1) OS posterior mean $\mathrm{HR} \leq 0.8$ with probability better $\geq 80 \%$ as compared to interferon, (2) PFS posterior mean HR $\leq 0.6$ with probability better than interferon $\geq 90 \%$, and (3) ORR posterior mean OR $>3.0$ with probability better than interferon $\geq 95 \%$ were deemed to have a meaningful benefit compared to interferon [13].

For this analysis, prior distributions on the hazard and odds ratios for each therapy relative to interferon within endpoint, between endpoints, and intra-cluster correlations were set uninformatively, but in a manner to ensure posterior sampling occurred in sensible parameter ranges. Prior distributions on the within study variances of each reported endpoint were taken to be centered at the reported (or recalculated from a confidence interval) value with variance inversely proportional to the number of subjects in the associated group. Posterior sampling was performed via 10,000 iterations of Markov chain Monte Carlo (MCMC), with the first 5,000 discarded as burn-in. Analyses were performed in R 3.4.0, with MCMC performed in Stan 2.17.0 called via rstan [14-16].

\section{RESULTS}

Table 1 summarizes the patient population, study arms, and relative efficacy measures in the 7 clinical trials included in the network meta-analysis. Metaestimates for OS and PFS hazard ratios as compared to interferon along with $95 \%$ credible intervals are shown in Fig. 1. Table 2 summarizes results from 
Overall Survival

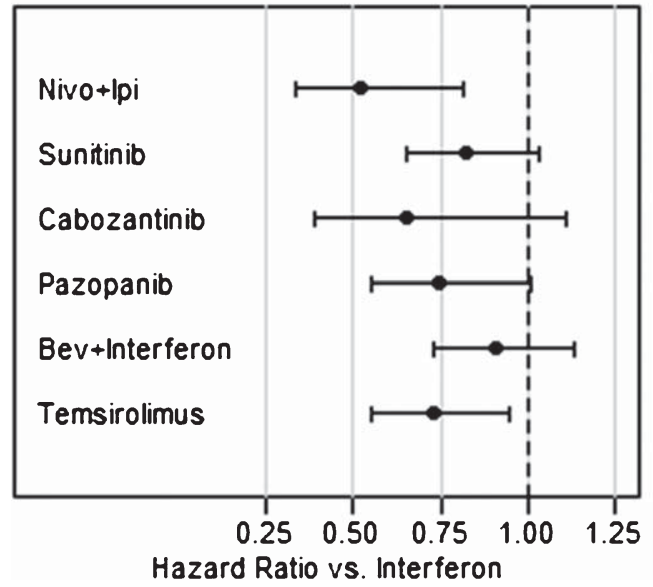

Progression-Free Survival

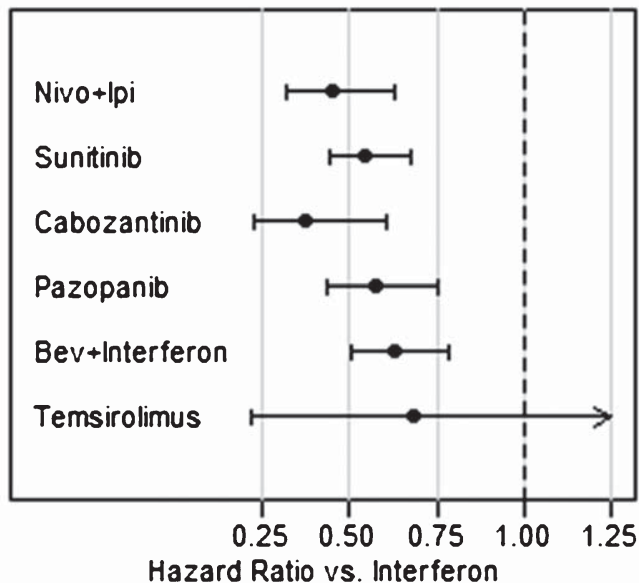

Fig. 1. Hazard ratios for first-line mRCC therapies as compared to interferon.

Table 1

Synopsis of first-line treatments included in network meta-analysis

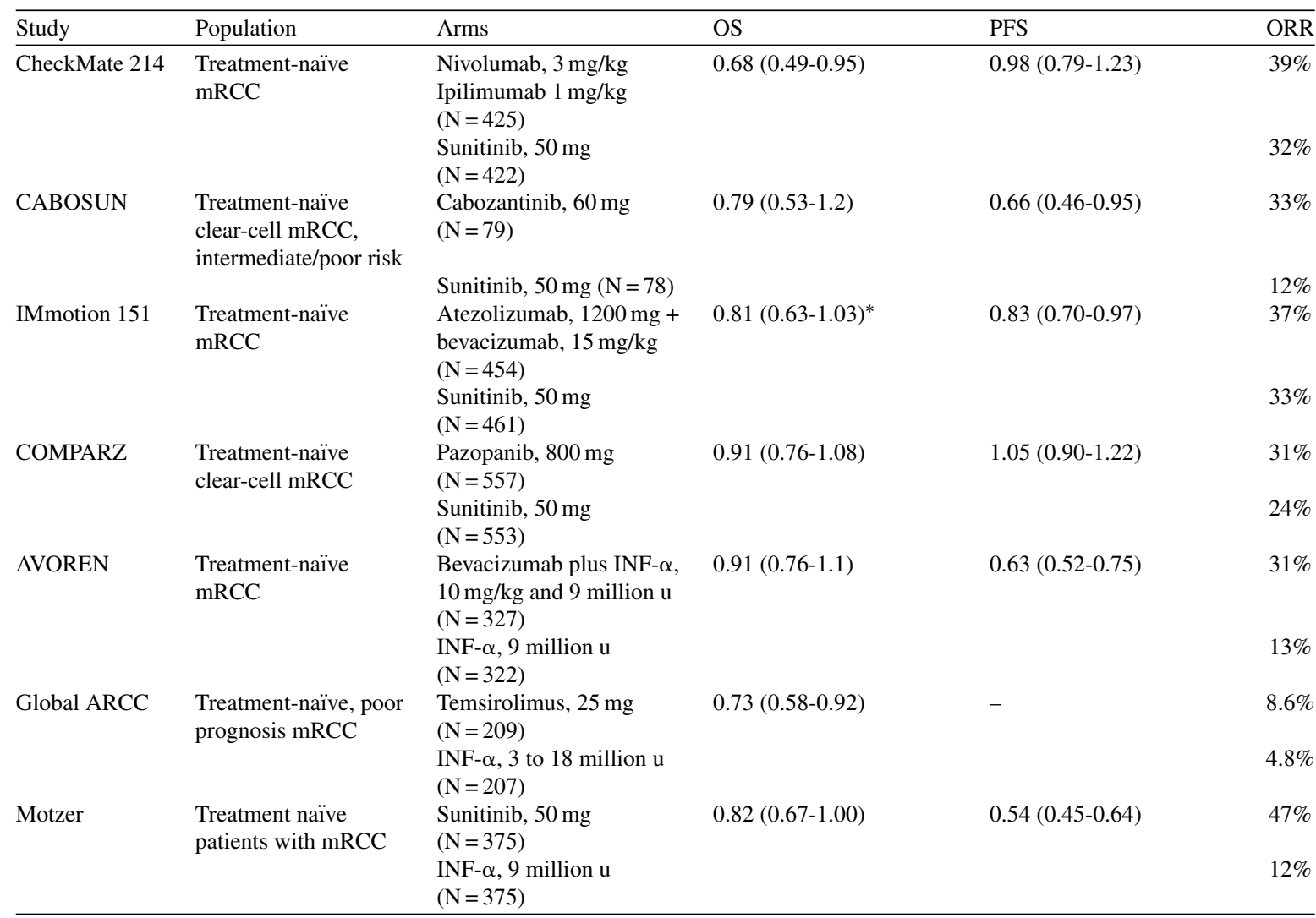

$\mathrm{OS}=$ overall survival, $\mathrm{PFS}=$ progression-free survival, $\mathrm{ORR}=$ objective response rates, $\mathrm{mRCC}=$ metastatic renal cell carcinoma, ${ }^{*}=\mathrm{OS}$ data from IMmotion 151 are immature, only $29 \%$ of patients had an OS event at data cutoff. 
Table 2

Efficacy summaries for first-line advanced or metastatic RCC therapies

\begin{tabular}{|c|c|c|c|c|c|c|c|c|c|}
\hline & OS & & & PFS & & & ORR & & \\
\hline Therapy & HR vs. INF- $\alpha$ & $\begin{array}{l}\text { Posterior } \\
\text { probability } \\
\text { better than } \\
\text { INF- } \alpha\end{array}$ & $\begin{array}{l}\text { Posterior } \\
\text { probability } \\
\text { therapy is best }\end{array}$ & HR vs. INF- $\alpha$ & $\begin{array}{l}\text { Posterior } \\
\text { probability } \\
\text { better than } \\
\text { INF- } \alpha\end{array}$ & $\begin{array}{l}\text { Posterior } \\
\text { probability } \\
\text { therapy is best }\end{array}$ & OR vs. INF- $\alpha$ & $\begin{array}{l}\text { Posterior } \\
\text { probability } \\
\text { better than } \\
\text { INF- } \alpha\end{array}$ & $\begin{array}{l}\text { Posterior } \\
\text { probability } \\
\text { therapy is best }\end{array}$ \\
\hline $\begin{array}{l}\text { Nivolumab + } \\
\text { Ipilimumab }\end{array}$ & $0.57(0.36-0.88)$ & $99 \%$ & $52 \%$ & $0.54(0.39-0.73)$ & $>99 \%$ & $2 \%$ & $\begin{array}{l}8.33 \\
(4.79-14.10)\end{array}$ & $>99 \%$ & $2 \%$ \\
\hline Cabozantinib & $0.63(0.39-1.05)$ & $97 \%$ & $27 \%$ & $0.37(0.24-0.57)$ & $>99 \%$ & $69 \%$ & $\begin{array}{l}20.14 \\
(7.82-54.09)\end{array}$ & $>99 \%$ & $95 \%$ \\
\hline $\begin{array}{l}\text { Bevacizumab + } \\
\text { Atezolizumab }\end{array}$ & $0.66(0.47-0.94)$ & $99 \%$ & $13 \%$ & $0.45(0.35-0.60)$ & $>99 \%$ & $13 \%$ & $\begin{array}{l}7.34 \\
(4.23-12.70)\end{array}$ & $>99 \%$ & $1 \%$ \\
\hline Temsirolimus & $0.73(0.55-0.94)$ & $99 \%$ & $6 \%$ & $0.68(0.22-2.34)$ & $73 \%$ & $16 \%$ & $2.08(0.88-4.99)$ & $95 \%$ & $<1 \%$ \\
\hline Pazopanib & $0.74(0.55-0.99)$ & $98 \%$ & $1 \%$ & $0.57(0.45-0.74)$ & $>99 \%$ & $<1 \%$ & $\begin{array}{l}8.81 \\
(5.14-14.63)\end{array}$ & $>99 \%$ & $2 \%$ \\
\hline Sunitinib & $0.82(0.65-1.02)$ & $96 \%$ & $<1 \%$ & $0.54(0.45-0.66)$ & $>99 \%$ & $<1 \%$ & $6.17(3.98-9.42)$ & $>99 \%$ & $<1 \%$ \\
\hline $\begin{array}{l}\text { Bevacizumab + } \\
\text { INF- } \alpha\end{array}$ & $0.91(0.73-1.11)$ & $81 \%$ & $<1 \%$ & $0.63(0.51-0.77)$ & $>99 \%$ & $<1 \%$ & $3.05(1.91-4.82)$ & $>99 \%$ & $<1 \%$ \\
\hline
\end{tabular}

OS = overall survival, $\mathrm{PFS}=$ progression-free survival, $\mathrm{ORR}=$ objective response rates, $\mathrm{mRCC}=$ metastatic renal cell carcinoma, $\mathrm{INF}-\alpha=$ interferon-alfa. 
the meta-analysis, including posterior median hazard ratios along with 95\% credible intervals as compared to interferon, posterior probabilities that each therapy is better than interferon, and posterior probabilities that the therapy is the best among those compared.

For first-line treatment of mRCC, cabozantinib may be superior to other agents for PFS (probability best $69 \%$ ) and ORR (probability best $95 \%$ ). In regards to OS, the data suggests that nivolumab plus ipilimumab may be better than comparators, but the strength of evidence is less compelling (probability best 52\%). By pre-specified criteria, there is no single treatment that is superior to other first-line treatments across all endpoints. Nivolumab plus ipilimumab, cabozantinib, bevacizumab plus atezolizumab, and pazopanib achieved meaningful benefit as compared to interferon.

\section{DISCUSSION}

First-line treatment of mRCC continues to evolve at a staggering pace. Most recently, pivotal phase 2 and 3 clinical trials were reported for nivolumab plus ipilimumab, cabozantinib, and bevacizumab plus atezolizumab. The standard of care arm in all three clinical trials was sunitinib, thus we do not have head-to-head comparisons to guide treatment selection among first-line agents. In the first indirect comparison of contemporary first-line treatments for mRCC, we found that no single treatment was superior across all endpoints. Cabozantinib may be superior to other agents for PFS and ORR, whereas, nivolumab plus ipilimumab may be the best treatment for OS. In sum, first-line treatment should be tailored to individual cases based on the unique characteristics of each treatment regimen.

In 2017, cabozantinib was approved for first-line treatment of mRCC on the basis of the CABOSUN clinical trial. CABOSUN was a phase 2 clinical trial that randomized 157 patients with intermediate or poor-risk, untreated, clear cell $\mathrm{mRCC}$ to cabozantinib or sunitinib [10]. In this trial, cabozantinib significantly improved PFS, OS, and ORR compared to sunitinib (Table 1). In our meta-analysis, evidence suggested first-line cabozantinib may be superior to other agents for PFS and ORR, so cabozantinib may be an ideal treatment for patients with rapidly progressive disease who need an objective response to treatment. While outcome by location of metastasis is not reported in CABOSUN or in this meta-analysis, a subgroup analysis from the clinical trial for salvageline cabozantinib, METEOR, found that patients with bone metastases have superior response to cabozantinib [17]. Hence, cabozantinib may also be an ideal therapy for patients with bone metastases. In regards to toxicity, cabozantinib is well tolerated and has a similar toxicity profile to other VEGF-TKIs, including diarrhea $(10 \%)$, treatment-related hypertension (28\%), and palmar-plantar erythrodysesthesia (8\%). To date, first-line cabozantinib has not been studied in International mRCC Database Consortium (IMDC) favorable-risk patients, which limits the population that cabozantinib should be recommended to. However, salvage-line cabozantinib was efficacious in IMDC favorable-risk patients, so it would not be surprising if first-line cabozantinib were also efficacious in this population. Finally, combinations of first-line cabozantinib and PD-1 axis inhibitors are an intriguing possibility. Clinical trials for these combinations are already underway, such as a phase 3 trial of first-line cabozantinib with nivolumab (NCT03141177).

Among first-line treatments for mRCC, nivolumab plus ipilimumab may be the best in regards to OS (Table 2). The combination of nivolumab, a PD1 inhibitor, plus ipilimumab, a CTLA-4 inhibitor, was initially approved for treatment of metastatic melanoma [18]. While not currently approved for the treatment of mRCC, nivolumab plus ipilimumab will likely be approved for first-line treatment on the basis of CheckMate 214. CheckMate 214 was a phase 3 clinical trial that randomized 1096 treatmentnaïve $\mathrm{mRCC}$ patients to nivolumab plus ipilimumab or sunitinib [11]. The combination met the coprimary endpoints of improved OS and ORR in intermediate and poor-risk patients. For the purpose of our meta-analysis, we used the intention-totreat cohort from CheckMate 214 to provide an equal comparison across clinical trials (Table 1). In comparison to cabozantinib, nivolumab plus ipilimumab is notable for having a superior complete response rate ( $9 \%$ vs. $1 \%$ ). However, the toxicity profile of nivolumab plus ipilimumab differs significantly from TKIs and nivolumab as monotherapy. Of all patients treated with nivolumab plus ipilimumab, $60 \%$ required systemic corticosteroids for management of an immune-related adverse event. In metastatic melanoma and mRCC, combination immunotherapy has resulted in significantly higher rates of immune-related adverse events compared to PD-1 inhibitor monotherapy. 
In our meta-analysis, none of the remaining firstline treatments for $\mathrm{mRCC}$ were superior for any efficacy endpoint. However, bevacizumab plus atezolizumab offers a promising combination of efficacy and tolerability. Bevacizumab plus atezolizumab is the first VEGF targeted therapy and immunotherapy combination to be studied in a phase 3 clinical trial, IMmotion 151. In that trial, the combination of bevacizumab plus atezolizumab improved PFS and ORR compared to sunitinib (Table 1) [12]. Similarly to nivolumab plus ipilimumab, bevacizumab plus atezolizumab has superior complete remission rates compared to sunitinib (5\% by investigator assessment, $11 \%$ by independent review committee). In contrast to nivolumab plus ipilimumab, bevacizumab plus atezolizumab was better tolerated than sunitinib (grade 3/4 adverse events $40 \%$ vs. 54\%). Among other first-line treatment options available for $\mathrm{mRCC}$, we expect that first-line use of single agent bevacizumab or temsirolimus will become less prevalent. Finally, we anticipate that the treatment landscape for mRCC will continue to evolve as the results from phase 3 clinical trials evaluating novel combinations of VEGF targeted therapy and immunotherapy are reported [19].

The findings from our network meta-analysis are limited by the design of multiple clinical trials included in our analysis. The CABOSUN trial was the only phase 2 clinical trial included in our analysis. Furthermore, it only included IMDC intermediate and poor-risk patients. In an attempt to provide a similar comparison across the remaining trials, we used the intention-to-treat analyses from CheckMate 214 and IMmotion 151. The primary endpoints for both of these trials were for specific subgroups of patients. Finally, the OS data from IMmotion 151 is still immature, so it may change with future updates from the trial.

\section{CONCLUSION}

In the first indirect comparison of contemporary first-line treatments for mRCC, evidence suggests cabozantinib may be superior to other agents for PFS and ORR, whereas, nivolumab plus ipilimumab may be best treatment for OS. No single agent was superior to comparators across all endpoints. First-line treatment for mRCC should be tailored to individual cases based off the unique characteristics of each treatment regimen. We anticipate the treatment landscape for $\mathrm{mRCC}$ will continue to change over the next five years as clinical trials evaluating combination therapy are reported.

\section{ACKNOWLEDGEMENTS}

\section{Conflict-of-interest statement}

AWH, PH, and $\mathrm{BH}$ have no conflict-of-interest to disclose. Neeraj Agarwal reports consultancy to Pfizer, Novartis, Merck, Genentech, Eisai, Exelixis, Clovis, and EMD Serono.

\section{Funding}

No funding supported this manuscript.

\section{Authorship contribution}

AWH, NA, and BH contributed to the design and conception of this study. AWH and PH collected all clinical data. BH performed all statistical analyses. AWH and PH wrote the initial draft. NA, BH, BLM, and AWH performed critical revisions.

\section{REFERENCES}

[1] Siegel RL, Miller KD, Jemal A. Cancer statistics, 2018. CA Cancer J Clin. n/a-n/a.

[2] Motzer RJ, Bander NH, Nanus DM. Renal-cell carcinoma. N Engl J Med. 1996;335:865-75.

[3] Janzen NK, Kim HL, Figlin RA, Belldegrun AS. Surveillance after radical or partial nephrectomy for localized renal cell carcinoma and management of recurrent disease. Urol Clin North Am. 2003;30:843-52.

[4] Ko JJ, Xie W, Kroeger N, Lee JL, Rini BI, Knox JJ, et al. The International Metastatic Renal Cell Carcinoma Database Consortium model as a prognostic tool in patients with metastatic renal cell carcinoma previously treated with first-line targeted therapy: A population-based study. Lancet Oncol. 2015;16:293-300.

[5] Escudier B, Eisen T, Stadler WM, Szczylik C, Oudard S, Siebels M, et al. Sorafenib in advanced clear-cell renal-cell carcinoma. N Engl J Med. 2007;356:125-34.

[6] Motzer RJ, Hutson TE, Tomczak P, Michaelson MD, Bukowski RM, Rixe O, et al. Sunitinib versus interferon alfa in metastatic renal-cell carcinoma. N Engl J Med. 2007;356:115-24.

[7] Sternberg CN, Davis ID, Mardiak J, Szczylik C, Lee E, Wagstaff J, et al. Pazopanib in locally advanced or metastatic renal cell carcinoma: Results of a randomized phase III trial. J Clin Oncol. 2010;28:1061-8.

[8] Escudier B, Pluzanska A, Koralewski P, Ravaud A, Bracarda $\mathrm{S}$, Szczylik C, et al. Bevacizumab plus interferon alfa-2a for treatment of metastatic renal cell carcinoma: A randomised, double-blind phase III trial. Lancet. 2007;370:2103-11.

[9] Hudes G, Carducci M, Tomczak P, Dutcher J, Figlin $\mathrm{R}$, Kapoor A, et al. Temsirolimus, interferon alfa, or both for advanced renal-cell carcinoma. N Engl J Med. 2007;356:2271-81 
[10] Choueiri TK, Halabi S, Sanford BL, Hahn O, Michaelson MD, Walsh MK, et al. Cabozantinib versus sunitinib as initial targeted therapy for patients with metastatic renal cell carcinoma of poor or intermediate risk: The alliance A031203 CABOSUN trial. J Clin Oncol. 2017;35: 591-7.

[11] Escudier B, Tannir, N., McDermott, DF, et al. CheckMate 214: Efficacy and safety of nivolumab + ipilimumab $(\mathrm{N}+\mathrm{I})$ $\mathrm{v}$ sunitinib (S) for treatment-naïve advanced or metastatic renal cell carcinoma (mRCC), including IMDC risk and PD-L1 expression subgroups. Ann Oncol. 2017;28.

[12] Motzer RJ, Powles T, Atkins MB, et al. IMmotion151: A randomized phase III study of atezolizumab plus bevacizumab vs sunitinib in untreated metastatic renal cell carcinoma (mRCC). J Clin Oncol. 2018;36:Abstr 578.

[13] Salanti G, Ades AE, Ioannidis JP. Graphical methods and numerical summaries for presenting results from multipletreatment meta-analysis: An overview and tutorial. J Clin Epidemiol. 2011;64:163-71.
[14] Team RC. R: A Language and Environment for Statistical Computing. 2017.

[15] Team SD. Stan modeling language users guide and reference manual. 2017;2.17.0.

[16] Team SD. RStan: The R interface to Stan. 2017;R package version 2.16.2.

[17] Escudier B, Powles T, Motzer RJ, Olencki T, Aren Frontera O, Oudard S, et al. Cabozantinib, a new standard of care for patients with advanced renal cell carcinoma and bone metastases? Subgroup Analysis of the METEOR Trial. J Clin Oncol. 2018:Jco2017747352.

[18] Larkin J, Chiarion-Sileni V, Gonzalez R, Grob JJ, Cowey $\mathrm{CL}$, Lao $\mathrm{CD}$, et al. Combined nivolumab and ipilimumab or monotherapy in untreated melanoma. $\mathrm{N}$ Engl $\mathrm{J}$ Med. 2015;373:23-34.

[19] Gill DM, Hahn AW, Hale P, Maughan BL. Overview of current and future first-line systemic therapy for metastatic clear cell renal cell carcinoma. Curr Treat Options Oncol. 2018;19:6. 